\title{
ABDOMINAL ANEURISM WHICH RUPTURED THROUGH THE DIAPHRAGM.
}

\author{
BY HENRY GRAY CROLY, F.R.C.S.; \\ Senior Surgeon, City of Dublin Hospital ; \\ Past President, Royal College of Surgeons :
}

AND

\author{
W IL L I M R. GRAVES, M. D.; \\ Pathologist to the City of Dublin Hospital ; \\ Member, British Institute of Public Health.
}

[Read before the Pathological Section, May 17, 1895.]

J. O'N., aged thirty-five years, bodymaker for Dublin, Wicklow, and Wexford Railway, was admitted to the City of Dublin Hospital on March 27 th, 1895, complaining of a pain in the loins, chiefly in the region of the left kidney. The pain, which was severe, he first felt about five months previously; but it ceased until about a month before his admission into hospital, and of late he lost flesh considerably.

On admission he was very anæmic, and had an anxious expression. On examination the lower end of the left kidney could be felt, the size being normal; there was no blood in the urine. The treatment consisted of perfect rest in bed, dry cuppings over the loins, and the application of hot-water bags, enemata, and careful regime, with occasional sedatives, also iodide and bromide of potassium. No tumour of any sort could be discovered by palpation, except the projecting kidney. In my cliniques I said the kidney, though easily felt, was apparently normal in size, and seemed to be pushed down. There was aibumen in the urine.

On May 1 st the patient was attacked by very severe pains down both legs, the pain coming on in spasms, and following the branches of the anterior crural nerve. Bromide draughts gave him ease, and he slept fairly well during the night.

The following morning (May 2nd) he seemed better, and said all pain had left him. He got out of bed at about 430 o'clock, and was speaking to the patients in the ward. He went back to bed again at about 5 o'clock, took a good tea, and was speaking to 
the nurse at 530 p.m., apparently quite well. At 6 o'clock he rose in the bed for a moment, groaned, and fell back and expired in a few minutes.

The diagnosis of abdominal aneurism is a matter of great difficulty, and the disease is more frequently discovered after death of the patient than detected, or even suspected, during life. The symptoms frequently resemble lumbagn, intestinal trouble, rheumatic gout, or malignant disease of the viscera; and it is only when these tumours can be recognised by the senses of sight and touch, assisted by auscultation, that anything approaching a correct diagnosis can be made.

Autopsy, by Dr. Graves.-Made a left lumbar colotomy incision, and found the left kidney displaced downwards, and felt the lower edge of a tumour, which afterwards proved to be an aneurism of abdominal aorta. Placed the body on its back, and made the usual median incision. On putting in the knife at the second costal cartilage, about ten ozs. of blood-stained serum escaped. On opening the thorax, the left pleura was found to be quite full of clotted blond, the left lung having collapsed. On removing the lung and blood clot from the left thorax, it was possible to pass the fingers through the diaphragm into the sac of the aneurism which was in the abdomen. On opening the abdomen, the spleen was found to be a little enlarged, and displaced downwards and forwards. On removing the stomach, spleen, and bowels, the sac of the aneurism came into view. The aneurism sprang from the abdominal aorta posterior to the coliac axis, and was firmly adherent to 12th dorsal and 1st and 2nd lumbar vert. The sac of the aneurism was also firmly adherent to the left psoas muscle and to the diaphragm. On laying open the aorta from the front, the neck of the sac was very plainly seen.

Kidneys.-Slightly granular and fatty.

Heart.-Atheromatous patches on mitral valve. Patent foramen ovale arch of aorta very atheromatous.

Lungs.-Congested and venous-looking.

Dr. M'W Eeney wished to know the mechanism by which an aneurism situated behind the coeliac axis ruptured into the left 
By Mr. H. G. Croly and Dr. Graves. $3: 1$

pleural cavity. In the section of the kidney there was only a small part which showed increased connective tissue, with a few atrophied glomeruli. He did not consider that sufficient to make it a granular kidney. The kidney also presented a smooth surface. A short time ago he saw a case in which an aneurism, situated in the middle of the dorsal region of the aorta, had ruptured into the substance of the lung and not into the pleural cavity. The man fell dead, and blood gushed from his mouth and nose.

Dr. Croly said that the man was admitted and treated for kidney disease. The only explanation he could give of the absence of pulsation was, that the tumour was bound down by very strongr adhesions.

Dr. Graves, in reply to Dr. M'Weeney, said that the aneurism had become glued to the psoas muscle, and had eroded it and shoved it up, and that it had then burst through the diaphragm, $\mathrm{He}$ agreed with him that the kiduey only showed interstitial changes in one part. 